\title{
Accuracy of saline infusion sonography versus hysteroscopy in the evaluation of uterine cavity abnormalities in infertile women at CHRACERH, Yaounde, Cameroon
}

\author{
Claude C. Noa Ndoua ${ }^{1,2 *}$, Etienne Belinga ${ }^{1,2}$, Gaspard G. Ayissi Ngah $^{1}$, Junie N. Metogo ${ }^{1,2}$, \\ Sandrine Mendibi ${ }^{2}$, Jean Marie Kasia ${ }^{1,2}$
}

\author{
${ }^{1}$ Department of Gynecology, Faculty of Medicine, University of Yaounde 1, Cameroon \\ ${ }^{2}$ Department of Obstetrics and Gynecology, Endoscopic Surgery and Human Reproductive Teaching Hospital, \\ Yaounde, Cameroon
}

Received: 29 August 2018

Accepted: 27 September 2018

\author{
*Correspondence: \\ Dr. Claude C. Noa Ndoua, \\ E-mail: claudenoa@yahoo.co.uk
}

Copyright: () the author(s), publisher and licensee Medip Academy. This is an open-access article distributed under the terms of the Creative Commons Attribution Non-Commercial License, which permits unrestricted non-commercial use, distribution, and reproduction in any medium, provided the original work is properly cited.

\begin{abstract}
Background: Uterine cavity abnormalities are seen as a cause of infertility in around 10\%-15\% and can go up to $50 \%$ in women with recurrent implantation failure. Saline infusion sonohysterography (SIS) is a minimally invasive diagnostic modality in the evaluation but hysteroscopy remains the gold standard in the assessment of intra uterine cavity. The aim of this study was to compare the diagnostic accuracy of saline infusion sonography (SIS) considering hysteroscopy (HS) as the gold standard to evaluate uterine cavity pathologies in infertile women at CHRACERH.

Methods: Authors carried out a cross-sectional, retrospective study, over 2 years, from the 1st January 2016 to the 31st December 2017, with retrospective data collection in 110 records of infertile patients subjected to an ultrasound assessment of uterine cavity using saline as the contrast medium with a $6.5-\mathrm{MHz}$ transvaginal transducer. When SIS found intra uterine abnormality, HS was done on a later date, using a $5 \mathrm{~mm}$ Bettocchi hysteroscope. Sensitivity, specificity, accuracy, positive and negative predictive values of SIS and HS were compared using the SPSS 20 software.

Results: The mean age and body mass index were respectively $39.3 \pm 7.8$ years and $28.7 \pm 4.1 \mathrm{~kg} / \mathrm{m}^{2}$. The main findings both in SIS and Hysteroscopy were respectively polyps $(\mathrm{n}=61 ; 55.5 \%$ vs $52.7 \%$; $\mathrm{n}=58)$, myomas $(\mathrm{n}=43 ; 39.1 \%$ vs $31.8 \% ; n=35)$, intrauterine adhesions $(n=18 ; 16.4 \%$ vs $21.8 \% ; n=24)$. The overall sensitivity, specificity and accuracy of SIS were $81.2 \%, 86.9 \%$ and $86.5 \%$ respectively.

Conclusions: SIS as a diagnostic tool in the evaluation of intrauterine lesions has a good accuracy and can therefore replace HS when this later is not available, especially in our African setting.
\end{abstract}

Keywords: Hysteroscopy, Saline infusion sonography, Sensitivity, Specificity, Uterine cavity abnormalities

\section{INTRODUCTION}

Uterine cavity abnormalities are seen as a cause of infertility in around $10 \%-15 \%$ of women. In women with recurrent implantation failure abnormalities are found in up to $50 \%$ of the women. ${ }^{1}$ Evaluation of the uterine cavity is a basic step in the investigation of infertile women because the most critical step for successful IVF outcome is embryo implantation, which is influenced by a positive cross talk between an adequate quality embryo and a receptive endometrium. ${ }^{2}$

Any uterine pathologies such as polyps, myoma, adhesions or congenital malformation can therefore interfere with the implantation process. ${ }^{3}$ Thus, it is recommended to diagnose and treat these abnormalities 
as their surgical correction may potentially improve the prospects of conception and a subsequent successful pregnancy. 4

Although transvaginal ultrasound is the most common diagnostic tool used to evaluate the regularity and shape of the uterine cavity, its diagnostic accuracy is poor with pathologies like polyps, adhesions, and submucous fibroids being missed. Recent studies have reported poor sensitivity and positive predictive value (PPV) rates of transvaginal sonography (TVS) in the detection of polypoid lesions. $^{5}$

Saline infusion sonography (SIS) is a diagnostic technique with many advantages. It is performed in a comparatively shorter duration of time, is more cost effective, and is less painful for patients than HS, but hysteroscopy remains the gold standard procedure for uterine cavity assessment providing a real time view, and allowing immediate treatment possible, although it is a costly invasive procedure associated with its share of discomfort and risks. ${ }^{6}$ Reports on the diagnostic accuracy of saline infusion sonography are conflicting. Some authors consider a negative saline contrast hysterosonography as proof of a normal uterine cavity, whereas others report insufficient diagnostic accuracy to rely on in clinical practice. ${ }^{1,7}$

Therefore, authors sought to compare the accuracy of SIS versus HS in the assessment of uterine cavity abnormalities in infertile women, for the first time in an African population at the Hospital Center for Research and Application in Endoscopic Surgery and Human Reproduction (CHRACERH) in Yaounde, Cameroon.

\section{METHODS}

Authors carried out a cross-sectional, retrospective study, over 2 years, from the $1^{\text {st }}$ January 2016 to the $31^{\text {st }}$ December 2017. The study was done through 4 months period, from the $1^{\text {st }}$ September to the $31^{\text {st }}$ December 2017 , at the Hospital Center for Research and Application in Endoscopic Surgery and Human Reproduction (CHRACERH) in Yaounde, Cameroon.

Data (age, body mass index, past medical history, SIS findings, indications of hysteroscopy, hysteroscopic findings) were collected from the records of patients undergoing diagnostic or operative hysteroscopy for several indications at CHRACERH. An authorization was obtained beforehand from the CHRACERH ethics committee.

Patients: 110 records of infertile women were enrolled in this study. Patients underwent an ultrasound assessment of the uterine cavity with saline as the contrast medium (SIS) and then a HS was performed to visualize the intrauterine anomaly. SIS was performed after the menstrual cycle, during the proliferative phase of the menstrual cycle, i.e. between the $6^{\text {th }}$ and the $11^{\text {th }}$ day for a 28-day cycle using a $6.5-\mathrm{MHz}$ transvaginal transducer. The patient was placed in the lithotomy position. A sterile speculum was placed into the vagina and the cervix was brought into view. The cervix was then cleansed with a povidone-iodine solution. The pediatric Foley's catheter and stiffener were placed at the external cervical os and the catheter was advanced through the stiffener into the endometrial canal; the balloon was inflated with $2 \mathrm{ml}$ saline so that the catheter did not become dislodged. The speculum was removed carefully and the endovaginal probe was reinserted beside the catheter. Under direct sonographic visualization, the balloon was gently retracted to occlude the internal cervical os. Again, under sonographic guidance, $\sim 5-20 \mathrm{ml}$ of warm sterile saline was injected, sonographic evaluation of the endometrial cavity was performed in both the coronal and the sagittal planes, the balloon was then deflated, and evaluation of the lower uterine segment and the endocervical region was performed. All the SIS were done in external medical centers by different qualified radiologists

Hysteroscopy was done at CHRACERH during the follicular phase of the menstrual cycle, i.e. between the 6 th and the 11th day for a 28-day cycle. Patients were in the gynecological position in the operating room under rachianaesthesia. After cleaning the external genitalia, vagina and cervix with an antiseptic solution (10\% povidone-iodine), sterile fields were placed; the first step consisted of diagnostic hysteroscopy with a Bettocchi (a continuous flow panoramic rigid hysteroscope, $26 \mathrm{~cm}$ in length, $5 \mathrm{~mm}$ of outer diameter sheath and $0^{\circ}$ fibroptic lens Karl Storz Endoscopy, Utrecht, Netherlands). The distension of the uterine cavity was performed with normal saline prior to the uterine cavity evaluation. The second step consisted of an operative hysteroscopy if the indication was made. After dilation of the cervix with Hegar's candles, the resection of the polyps, myomas, synechiae or the sampling was done with the chisel or the resectoscope. All the samples removed were sent to the pathologist for analysis. First-step analgesics, nonsteroidal anti-inflammatory and levonorgestrelethinylestradiol tablets were given to the patients for the postoperative pain management and to increase endometrial thickness.

\section{Statistical analysis}

Statistical analysis was done using the SPSS 20 software. The sensitivity, specificity, accuracy, and the predictive values of the SIS and HS were calculated and compared using the following formulas:

- Sensitivity: probability that the test results will be positive when the disease is present (true positive rate, expressed as a \%);

- Specificity: probability that the test results will be negative when the disease is present (true negative rate, expressed as a \%);

- Positive predictive value (PPV: probability that the disease is present when the test is positive); 
- Negative predictive value (NPV: probability that the disease is present when the test is negative); and accuracy is the ratio of the true positive and true negative in all patients.

\section{RESULTS}

\section{Biophysical parameters}

Age and BMI. The mean age was $39.3 \pm 7.8$ years with a minimum at 21 years and a maximum at 75 years. The mean body mass index was $28.7 \pm 4.1$. Twenty per cent $(20 \%)$ of the study population was obese (BMI $>30$ $\left.\mathrm{kg} / \mathrm{m}^{2}\right)$.

Table 1: Distribution of the study population according to some biophysical parameters.

\begin{tabular}{|lllll|} 
Characteristic & $\mathbf{N}$ & $\begin{array}{l}\text { Mini- } \\
\text { mum }\end{array}$ & $\begin{array}{l}\text { Maxi- } \\
\text { mum }\end{array}$ & Mean \\
\hline Age & 110 & 21.0 & 75.0 & $39.7 \pm 7.8$ \\
\hline BMI & 110 & 19.8 & 39.6 & $28.7 \pm 4.1$ \\
\hline
\end{tabular}

\section{Hysterosonography versus hysteroscopy in the studied} population

The main findings both in SIS and Hysteroscopy were respectively polyps $(\mathrm{n}=61 ; 55.5 \%$ vs $52.7 \% ; \mathrm{n}=58)$, myomas $(\mathrm{n}=43 ; 39.1 \%$ vs $31.8 \% ; \mathrm{n}=35)$, intrauterine adhesions $(\mathrm{n}=18 ; 16.4 \%$ vs $21.8 \% ; \mathrm{n}=24)$ and septate uterus $(\mathrm{n}=2 ; 1.8 \%$ vs $2.7 \% ; \mathrm{n}=3)$.

\section{Diagnostic value for uterine myomas}

HS was more sensitive (100 vs $85 \%$ ), more specific (100 vs $80 \%)$, and more accurate $(100 \%$ vs $80 \%)$ than SIS in the evaluation of intracavitary myomas in the studied population. HS also had higher predictive value than SIS in the evaluation of intracavitary myomas (100 vs $65.1 \%$ PPV; 100 vs $89.5 \%$ NPV).

Table 2: Hysterosonography versus hysteroscopy in the studied population.

\begin{tabular}{|lll|}
\hline Variables & $\begin{array}{l}\text { Hysterosonography } \\
\text { n }(\%)\end{array}$ & $\begin{array}{l}\text { Hysteroscopy } \\
\text { n }(\%)\end{array}$ \\
\hline Polyps & $61(55.5)$ & $58(52.7)$ \\
\hline Myomas & $43(39.1)$ & $35(31.8)$ \\
\hline Synechiae & $18(16.4)$ & $24(21.8)$ \\
\hline $\begin{array}{l}\text { Bicorn uterus } \\
\text { or uterin } \\
\text { septum in } \\
\text { hysteroscopy) }\end{array}$ & $2(1.8)$ & $3(2.7)$ \\
\hline $\begin{array}{l}\text { Calcifications } \\
\text { Endometrial } \\
\text { thickening }\end{array}$ & $3(2.7)$ & $0(0)$ \\
\hline
\end{tabular}

\section{Diagnostic value for uterine polyps}

HS was more sensitive (100 vs $89.6 \%$ ), more specific (100 vs $82.6 \%$ ), and more accurate (100\% vs $86 \%$ ) than SIS in the evaluation of polyps in the studied population. HS also had higher predictive value than SIS in this purpose (100 vs $85 \%$ PPV; 100 vs $87 \%$ NPV).

\section{Diagnostic value for uterine synechiae}

For intrauterine adhesions, HS was more sensitive (100 vs $73.9 \%$ ), more specific (100 vs $98 \%)$, and more accurate $(100 \%$ vs $93.6 \%)$ than SIS.

HS also had higher predictive value than SIS in the evaluation of intrauterine adhesions in this population (100 vs $94.4 \%$ PPV; 100 vs $93.4 \%$ NPV).

Table 3: Diagnostic value for uterine myomas.

\begin{tabular}{|llllll|} 
& Sensitivity & Specificity & Positive predictive value & Negative predictive value Accuracy \\
\hline Hysterosonography & $80 \%$ & $80 \%$ & $65.1 \%$ & $89.5 \%$ & $80 \%$ \\
\hline Hysteroscopy & $100 \%$ & $100 \%$ & $100 \%$ & $100 \%$ & $100 \%$ \\
\hline
\end{tabular}

Table 4: Diagnostic value for uterine polyps.

\begin{tabular}{|llllll} 
& Sensitivity & Specificity & Positive predictive value & Negative predictive value & Accuracy \\
\hline Hysterosonography & $89.6 \%$ & $82.6 \%$ & $85 \%$ & $87 \%$ & $86 \%$ \\
\hline Hysteroscopy & $100 \%$ & $100 \%$ & $100 \%$ & $100 \%$ & $100 \%$ \\
\hline
\end{tabular}

Table 5: Diagnostic value for uterine synechiae.

\begin{tabular}{|llllll|} 
& Sensitivity & Specificity & Positive predictive value & Negative predictive value Accuracy \\
\hline Hysterosonography & $73.9 \%$ & $98 \%$ & $94.4 \%$ & $93.4 \%$ & $93.6 \%$ \\
\hline Hysteroscopy & $100 \%$ & $100 \%$ & $100 \%$ & $100 \%$ & $100 \%$ \\
\hline
\end{tabular}




\section{Overall diagnostic value of SIS versus HS}

In this study, the HS was more sensitive (100 vs $81.2 \%$ ), more specific (100 vs $86.9 \%$ ), and was more accurate
(100\% vs $86.5 \%)$ than SIS, and the HS also had higher predictive values (100 vs $81.5 \%$ PPV; 100 vs $90 \%$ NPV) than SIS in the evaluation of intracavitary lesions among infertile women.

Table 6: Overall diagnostic value of SIS versus HS.

\begin{tabular}{|l|lllll|}
\hline & Sensitivity & Specificity & Positive predictive value & Negative predictive value Accuracy \\
\hline Hysterosonography & $81.2 \%$ & $86.9 \%$ & $81.5 \%$ & $90 \%$ & $86.5 \%$ \\
\hline Hysteroscopy & $100 \%$ & $100 \%$ & $100 \%$ & $100 \%$ & $100 \%$ \\
\hline
\end{tabular}

\section{DISCUSSION}

In this study, the mean age which was $39.3 \pm 7.8$ years with a minimum at 21 years and a maximum at 75 years showed that present study population consisted in eldery infertile women. Indeed, advanced reproductive age is significantly associated with poor reproductive outcomes and infertility in these women may be the resultant of uterine factors, of the decrease of the ovarian reserve, of the alteration of tubal functions, or due to the higher rate of chromosomal abnormalities among them. ${ }^{1,8,9}$ On another side, the mean body mass index was $28.7 \pm 4.1$ $\mathrm{kg} / \mathrm{m}^{2}$, thus showing that present study population was overweight. The overweight here can be seen as another risk factor of infertility, when authors know that obesity and overweight have negative impact in reproductive health, including reduced pregnancy and live birth rates, increased miscarriage rates and pregnancy complications, both in natural and assisted conceptions. ${ }^{10}$

The main findings in both SIS and Hysteroscopy were respectively polyps $(\mathrm{n}=61 ; 55.5 \%$ vs $52.7 \% ; \mathrm{n}=58)$, myomas $(n=43 ; 39.1 \%$ vs $31.8 \% ; n=35)$ and intrauterine adhesions $(n=18 ; 16.4 \%$ vs $21.8 \% ; n=24)$. Similar data have been found by Pato-Mosquera et al in 904 patients undergoing diagnostic hysteroscopy after and ultrasound assessment of the uterine cavity; and those data suggest that polyps and myomas are overdiagnosed by SIS while intrauterine adhesions are underdiagnosed. ${ }^{11}$ This could be the fact of increased uterine mucosal folds which can be mistaken to small polyps or myomas by and ultrasound operator thus raising the operator-dependent nature of saline infusion sonography. In the same vein, mild synechiae may be missed at the SIS assessment as shown by Draz and colleagues in their study about 50 infertile patients undergoing SIS and HS. ${ }^{12}$

For diagnosing endometrial polyps, present study revealed sensitivity of $89.6 \%$ and a specificity of $82.6 \%$. The PPV, NPV and accuracy were $85 \%, 87 \%$ and $86 \%$ respectively. When evaluating submucous myomas, SIS showed sensitivity of $80 \%$ and specificity of $80 \%$. The PPV, NPV and accuracy were $65.1 \%, 89.5 \%$ and $80 \%$ respectively. Several studies have shown similar results in diagnosing polyps, submucous fibroids and hyperplasia. Tangri et al in their study about 136 infertile women found a sensitivity of $79 \%$ and a specificity of $89 \%$ for diagnosing polyp by SIS, whereas for diagnosis of submucous fibroids, sensitivity and specificity were $85 \%$ and $99 \%$ respectively. ${ }^{6}$ Nallapati et al found a sensitivity of $90.9 \%$ and a specificity of 92.7 for endometrial polyps while the sensitivity and the specificity for diagnosing submucous fibroids were $86.3 \%$ and $83 \%$ respectively. ${ }^{13}$ They all conclude that SIS can be a good alternative to HS with satisfying sensibility and specificity in the evaluation of intracavitary lesions. In this study, the specificity of SIS was comparable with the hysteroscopic one (98\% vs 100\%) for the diagnostic of intrauterine adhesions counterbalancing the relatively poor sensibility $(73.9 \%$ vs $100 \%)$, and its accuracy was the highest one in comparison with those of polyps and submucous myomas $(93.4 \%$ vs $86 \%$ and $80 \%$ respectively). Similar data have been found by Salle et al while Sitimani and col. found contradictories one. ${ }^{14,15}$ These finding show the high accuracy of the SIS in the diagnostic of intrauterine adhesions although mild synechiae may be missed at the SIS assessment.

Many studies have been carried out to compare SIS and HS in evaluating intrauterine abnormalities in several populations. ${ }^{6,12,13,16}$ The present study done for the first time in a sub-Saharan population, determined the overall sensitivity, specificity and accuracy to be $81.2 \%, 86.7 \%$ and $86.5 \%$ respectively. Similar data have been found by Draz et al with an overall sensitivity of $85 \%$, specificity of $100 \%$ and accuracy of $94 \% .{ }^{12}$ In the same vein, Tangri et al had both sensitivity and specificity at $88 \%$ in their study of 136 infertile women. ${ }^{6}$ The previous ones conclude that SIS can be a compromise alternative to HS when this later is not available, thus going in the same line of present African context where HS is still very rare. Summarizing, Seshadri et al in their meta-analysis found that the pooled sensitivity of SIS in the detection of all intrauterine abnormalities was 0.88 (95\% confidence interval (CI): $0.85-0.90)$. The pooled specificity was 0.94 (95\% CI 0.93-0.96). Hence, they conclude that SIS is a highly sensitive investigative modality and comparable to the gold standard tool, hysteroscopy in the detection of intrauterine abnormalities in sub fertile women. It is a highly sensitive and specific test in the diagnosis of 
uterine polyps, submucous myomas, uterine anomalies and intrauterine adhesions and can be used as a screening tool for subfertile patients prior to IVF treatment. ${ }^{16}$

The limitations of present study are the fact of the retrospective design of the study, and also the fact that the SIS have been performed in different medical centers by qualified radiologist. Although this can be a force by another point of view by reducing the operators bias and giving the true reality in present setting.

\section{CONCLUSION}

SIS as a diagnostic tool in the evaluation of intrauterine lesions has a good accuracy and can therefore replace HS when this later is not available, especially in present African setting.

\section{Funding: No funding sources}

Conflict of interest: None declared

Ethical approval: The study was approved by the CHRACERH Ethics Committee

\section{REFERENCES}

1. Pundir J, El Toukhy T. Uterine cavity assessment prior to IVF. Womens Health. 2010;6(6):841-8.

2. Van Voorhis BJ. Ultrasound assessment of the uterus and fallopian tube in infertile women. Semin Reprod Med. 2008;26(3):232-40.

3. Cakmak H, Taylor HS. Implantation failure: molecular mechanisms and clinical treatment. Hum Reprod Update. 2011;17(2):242-53.

4. Di Spiezio Sardo A, Di Carlo C, Minozzi S, Spinelli M, Pistotti V, Alviggi C, et al. Efficacy of hysteroscopy in improving reproductive outcomes of infertile couples: a systematic review and metaanalysis. Hum Reprod Update. 2016 J;22(4):479-96.

5. Bingol B, Gunenc Z, Gedikbasi A, Guner H, Tasdemir S, Tiras B. Comparison of diagnostic accuracy of saline infusion sonohysterography, transvaginal sonography and hysteroscopy. J Obstet Gynaecol. 2011;31(1):54-8.

6. Tangri MK, Srivastava AK. Diagnostic accuracy of saline infusion sonography as compared to hysteroscopy in premenopausal women with abnormal uterine bleeding. Int J Reprod Contracept Obstet Gynecol. 2017;6(2):682-7.

7. Bernard JP, Rizk E, Camatte S, Robin F, Taurelle R, Lecuru F. Saline contrast sonohysterography in the preoperative assessment of benign intrauterine disorders. Ultrasound Obstet Gynecol. 2001;17(2):145-9.

8. Amanvermez R, Tosun M. An Update on Ovarian Aging and Ovarian Reserve Tests. Int J Fertil Steril. 2016;9(4):411-5.

9. Refaat B, Dalton E, Ledger WL. Ectopic pregnancy secondary to in vitro fertilisation-embryo transfer: pathogenic mechanisms and management strategies. Reprod Biol Endocrinol. 2015;13(1):30.

10. Dağ ZÖ, Dilbaz B. Impact of obesity on infertility in women. J Turk Ger Gynecol Assoc. 2015;16(2):1117.

11. Pato-Mosquera M, Vázquez-Rodríguez M, PérezAdán M, García-García MJ, Blanco-Pérez S. Diagnostic hysteroscopy indications and results in Complexo Hospitalario Universitario De Ourense. Ginecol Obstet Mex. 2013;81(7):382-8.

12. Draz MH, El-Sabaa TM, Shorbagy SHE. Saline infusion sonography versus hysteroscopy in the evaluation of uterine cavity in women with unexplained infertility. Tanta Med J. 2017;45(3):155.

13. Nallapati S, Pallavee P, Ghose S. Comparative study of saline infusion sonography and hysteroscopy for evaluation of uterine cavity in abnormal uterine bleeding. Int J Reprod Contracept Obstet Gynecol. 2017;4(3):828-32.

14. Conforti A, Alviggi C, Mollo A, De Placido G, Magos A. The management of Asherman syndrome: a review of literature. Reprod Biol Endocrinol. 2013;11:118.

15. Sitimani A, Chawla I, Vohra P. Saline infusion sonography in evaluation of uterine cavity abnormalities in infertility: a comparative study. Int J Reprod Contracept Obstet Gynecol. 2017;5(9):29953000 .

16. Seshadri S, El-Toukhy T, Douiri A, Jayaprakasan K, Khalaf Y. Diagnostic accuracy of saline infusion sonography in the evaluation of uterine cavity abnormalities prior to assisted reproductive techniques: a systematic review and meta-analyses. Hum Reprod Update. 2015;21(2):262-74.

Cite this article as: Noa Ndoua $\mathrm{CC}$, Belinga E, Ngah GGA, Metogo JN, Mendibi S, Kasia JM. Accuracy of saline infusion sonography versus hysteroscopy in the evaluation of uterine cavity abnormalities in infertile women at CHRACERH, Yaounde, Cameroon. Int J Reprod Contracept Obstet Gynecol 2018;7:4355-9. 